\title{
Parameter Estimation for a Model With Both Imperfect Test and Repair
}

\author{
Simon Wilson ${ }^{1}$, Ben Flood ${ }^{1}$, Suresh Goyal ${ }^{2}$, Jim Mosher ${ }^{3}$, Susan Bergin ${ }^{2}$ \\ Joseph O'Brien ${ }^{4}$, Robert Kennedy ${ }^{4}$ \\ ${ }^{1}$ Centre for Telecommunications Value-Chain Research, Trinity College Dublin, Ireland \\ ${ }^{2}$ Bell Labs Ireland, Blanchardstown, Dublin 15, Ireland \\ ${ }^{3}$ Lucent Technologies, Columbus, Ohio, USA \\ ${ }^{4}$ Lucent Technologies, Blanchardstown, Dublin 15, Ireland \\ Email: swilson@tcd.ie; goyal@alcatel-lucent.com
}

\begin{abstract}
We describe estimation of the parameters of a manufacturing test and repair model using data available from that test. The model allows imperfect testing and imperfect repair. The principal problem that we address is of parameter identification, given insufficient data, that we address by making conservative assumptions on the property being measured and the associated parameter values. Several cases of commonly occurring test types, in the manufacture of electronic products, are considered.
\end{abstract}

\section{Introduction}

There is increasing interest in mathematical models for investigating and optimizing the manufacturing testing of electronic products, driven by the rapid increase and complexity in testing, and the fact that products being tested are composed of components from several suppliers and may be tested at globally distributed locations.

In this paper we propose an imperfect test and repair model, as in [1, 2], and develop methods for statistical estimation of the parameters of this model from test data. These estimations use common techniques, such as estimating a population mean and variance by a data sample mean and variance. This is done by defining a statistical model for the test measurements from which the test parameters are defined. Various measurement models are proposed, according to the type of test data observed; see Sections 3 to 6 . The test model remains the same and is described in Section 1.1. The test model parameters can be defined in terms of the measurement model parameters, and this is done in each of Sections 3 to 6 . There are complications because, for many tests, the data do not have sufficient information to estimate all the parameters uniquely. We address that by making sufficient assumptions about the parameter values of the measurement or test model, that we deem to be reasonable or conservative, so that unique estimates can be produced.

There are two novel contributions in this paper. First is its use of a statistical model for the test measurement data to implicitly define test parameters, so statistical estimation of test parameters from test data is possible. Second are the proposals for assumptions about the measurement or test model parameters in the cases where the test data are insufficient for their unique estimation.

\subsection{The Test Model}

In earlier papers [1,2], we developed mathematical models and a computational scheme for investigating the non-intuitive trade-offs between incurred test-costs and the resulting product quality (field performance, expected return rates, etc.) along the entire assembly, test and repair chain. The model parameter estimation described here is for various different test scenarios that are combinations of different property types being measured (continuous or discrete) and different data recorded (the measurement itself or simply whether the product passed or failed).

Modeling test and repair is an active research area. Early work on fault testing, such as [3], assumed perfect tests, which is unrealistic for electronic products. The optimal arranging of tests has also been investigated, for example [4]. Methods that allow false positive and false negative test results are more in line with our approach. Dislis et al. [5] have several papers in investigating the dynamics of test cost optimization, while in [6] a comprehensive model with imperfect testing and retesting is described. Trichy et al. [7] allow false positives as well as allowing defects to be introduced before and after test.

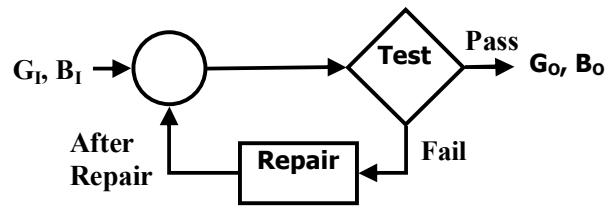

Fig. 1: Example Test \& Repair Network

Our model [1,2], illustrated with the simplest test and repair network in Fig. 1, is as follows. Assume that a property of a product is either good (i.e., it will not fail in the field during its expected life-time) or bad. These are tested imperfectly, with the chance of a false positive or a false negative result. These properties are measured during test; the measured value may be continuous or discrete. Those products that are measured to be bad are then sent for repair; the products measured bad are a mix of "truly" bad and "truly" good (the latter are the result of a false negative on the test). The repair process is also 
imperfect, and may not successfully repair a product. Repaired products are then re-tested, with the possibility as before of a false negative or a false positive result.

The test model is defined by the following parameters. We define $G_{I}$ to be the probability that a product is truly good (the proportion of good products). We define $\alpha_{\mathrm{BG}}$ to be the probability that a product that is truly bad is measured as good, or

$\alpha_{\mathrm{BG}}=\mathrm{P}($ product measured as good $\mid$ product is truly bad $)$. Similarly:

$\alpha_{\mathrm{GB}}=\mathrm{P}($ product measured bad $\mid$ product is truly good);

$\alpha_{\mathrm{BB}}=\mathrm{P}$ (product measured bad $\mid$ product is truly bad);

$\alpha_{\mathrm{GG}}=\mathrm{P}$ (product measured good $\mid$ product is truly good).

Since $\alpha_{\mathrm{GB}}=1-\alpha_{\mathrm{GG}}$ and $\alpha_{\mathrm{BG}}=1-\alpha_{\mathrm{BB}}$, it is sufficient to specify $\alpha_{\mathrm{GG}}$ and $\alpha_{\mathrm{BB}}$.

As we have said, the values of $\mathrm{G}_{\mathrm{I}}$ and the $\alpha$ 's are not defined explicitly in our model, although they are the quantities of interest. They are defined in terms of a statistical model for the value of the quantity being measured over the population of units, a statistical model for the measurement error of that value, and what makes a unit good or bad. It is the measurement error that leads us to make false positives and false negatives (so that $\alpha_{\mathrm{GB}}$ and $\alpha_{\mathrm{BG}}$ are not zero). As we have said, the advantage of this approach is that the test parameters can be estimated from data on the measurements recorded in the test.

We define $\beta$ 's to be the probabilities that truly $\operatorname{good} / \mathrm{bad}$ products are repaired to good/bad in the same way, e.g., $\beta_{\mathrm{GB}}=\mathrm{P}$ (product repaired to be bad $\mid$ product was truly good before), $\beta_{\mathrm{GG}}=\mathrm{P}$ (product repaired to be good $\mid$ product was truly good before), $\beta_{\mathrm{BB}}=\mathrm{P}$ (product repaired to be bad $\mid$ product was truly bad before) and $\beta_{\mathrm{BG}}$ $=\mathrm{P}($ product repaired to be good $\mid$ product was truly bad before). Since $\beta_{\mathrm{GB}}=1-\beta_{\mathrm{GG}}$ and $\beta_{\mathrm{BG}}=1-\beta_{\mathrm{BB}}$, it is sufficient to figure out $\beta_{\mathrm{GG}}$ and $\beta_{\mathrm{BB}}$. We will assume in all of this work that $\beta_{\mathrm{GB}}=0$, i.e., it is impossible to take a truly good product and, by repairing it, make it truly bad. This implies that $\beta_{\mathrm{GG}}=1$, leaving us to estimate only one free $\beta$ parameter (either $\beta_{\mathrm{BG}}$ or $\beta_{\mathrm{BB}}$ ).

\subsection{The Data}

We assume that there are three sets of data available. The first is a set that is collected only once from "one-off" experiments and is a set of $m$ repeated measurements $z_{1}, \ldots, z_{m}$ on one example of the product. These data have information on the size of the measurement error. The second set comes from every time that we test and is a set of measurements $y_{1}, \ldots, y_{n}$ on $n$ different examples of the product. We refer to this as the first pass data. The third set is the yield $p_{r G}$ on the second pass test (after repair), that is the proportion of units that pass this second run of the test.

\subsection{Structure of the Article}

The remainder of this paper is structured as follows. First, in Section 2, we describe how to estimate the $\beta$ 's were we to know the $\alpha^{\prime}$ s, $G_{I}$ and the yield of the second pass test of repaired items. We do this first because this is the same regardless of the measurement model. Sections 3 to 6 describe how to estimate the $\alpha$ 's and $G_{I}$ for 6 cases of data type and measurement that commonly occur in testing of electronic products. This is done by defining a statistical model for the data in each case and estimating the parameters of the statistical model using standard statistical estimation methods, and using these estimates to get values of $\mathrm{G}_{\mathrm{I}}$ and the $\alpha$ 's. Section 7 is a small case study and Section 8 concludes the paper with a discussion and conclusion.

\section{Estimating $\beta$ 's given $\alpha$ 's and $\mathbf{G}_{\mathbf{I}}$}

Assume that we observe the yield from the second test (the proportion of products passing the second test that failed the first pass test and were repaired), which we denote $p_{r G}$. This means that $\mathrm{p}_{\mathrm{rG}}$ is an estimate of the probability of passing the second pass test given that the first test is failed (product measured bad).

Assume for the moment that we have values for the $\alpha$ 's and $G_{I}$ (these will be obtained from the work described in sections 3, 4 and 5).

By the partition law:

$\mathrm{P}$ (failed 1st test)

$=\mathrm{P}($ failed 1 st test $\mid$ truly good $) \mathrm{P}$ (truly good $)$

$+\mathrm{P}($ failed 1 st test $\mid$ truly bad) $\mathrm{P}$ (truly bad)

$=\alpha_{\mathrm{GB}} G_{I}+\alpha_{\mathrm{BB}}\left(1-G_{I}\right)$

By Bayes' law and substituting in Eq. 2.1:

$\mathrm{P}$ (truly good before repair $\mid$ failed 1 st test)

$=\mathrm{P}($ failed 1 st test $\mid$ truly good $) \mathrm{P}($ truly good $)$ / P(failed 1st test)

$=\alpha_{\mathrm{GB}} G_{I} /\left(\alpha_{\mathrm{GB}} G_{I}+\alpha_{\mathrm{BB}}\left(1-G_{I}\right)\right)$

By the partition law and substituting in Eq. 2.2:

$\mathrm{P}$ (truly good after repair $\mid$ failed 1st test)

$=\mathrm{P}($ truly good after repair $\mid$ truly good before repair and failed 1st test)

$\times \mathrm{P}($ truly good before repair $\mid$ failed 1st test)

$+\mathrm{P}$ (truly good after repair | truly bad before repair and failed 1 st test)

$\times \mathrm{P}($ truly bad before repair $\mid$ failed 1 st test $)$

$=\left[\beta_{\mathrm{GG}} \alpha_{\mathrm{GB}} G_{I}+\beta_{\mathrm{BG}} \alpha_{\mathrm{BB}}\left(1-G_{I}\right)\right]$

$/\left[\alpha_{\mathrm{GB}} G_{I}+\alpha_{\mathrm{BB}}\left(1-G_{I}\right)\right]$

By the partition law again and substituting Eq. 2.3

we have:

$\mathrm{p}_{\mathrm{rG}} \approx \mathrm{P}$ (pass 2nd test $\mid$ failed 1st test $)$

$=\mathrm{P}($ product measured good $\mid$ truly good after repair and failed 1 st test)

$\times \mathrm{P}$ (truly good after repair $\mid$ failed 1st test)

$+\mathrm{P}$ (product measured good $\mid$ truly bad after repair and failed 1st test)

$\times \mathrm{P}($ truly bad after repair $\mid$ failed 1 st test $)$

$=\left[\alpha_{\mathrm{GG}} \beta_{\mathrm{GG}} \alpha_{\mathrm{GB}} G_{I}+\alpha_{\mathrm{GG}} \beta_{\mathrm{BG}} \alpha_{\mathrm{BB}}\left(1-G_{I}\right)\right.$

$\left.+\alpha_{\mathrm{BG}} \beta_{\mathrm{GB}} \alpha_{\mathrm{GB}} G_{I}+\alpha_{\mathrm{BG}} \beta_{\mathrm{BB}} \alpha_{\mathrm{BB}}\left(1-G_{I}\right)\right]$

$/\left[\alpha_{\mathrm{GB}} G_{I}+\alpha_{\mathrm{BB}}\left(1-G_{I}\right)\right]$.

Now substituting the assumptions that $\beta_{\mathrm{GB}}=0, \beta_{\mathrm{GG}}=$ 1 , and also $\beta_{\mathrm{BB}}=1-\beta_{\mathrm{BG}}$, we have: 


$$
\begin{gathered}
\mathrm{p}_{\mathrm{rG}} \approx\left[\alpha_{\mathrm{GG}} \alpha_{\mathrm{GB}} G_{I}+\alpha_{\mathrm{GG}} \beta_{\mathrm{BG}} \alpha_{\mathrm{BB}}\left(1-G_{I}\right)\right. \\
\left.+\alpha_{\mathrm{BG}}\left(1-\beta_{\mathrm{BG}}\right) \alpha_{\mathrm{BB}}\left(1-G_{I}\right)\right] \\
/\left[\alpha_{\mathrm{GB}} G_{I}+\alpha_{\mathrm{BB}}\left(1-G_{I}\right)\right]
\end{gathered}
$$

Re-arranging this equation gives a formula for $\beta_{\mathrm{BG}}$ :

$$
\beta_{B G}=\frac{p_{r G}\left(\alpha_{G B} G_{I}+\alpha_{B B}\left(1-G_{I}\right)\right)-\alpha_{G G} \alpha_{G B} G_{I}-\alpha_{B G} \alpha_{B B}\left(1-G_{I}\right)}{\alpha_{B B}\left(\alpha_{G G-} \alpha_{B G}\right)\left(1-G_{I}\right)} .
$$

The other $\beta$ 's are $\beta_{\mathrm{BB}}=1-\beta_{\mathrm{BG}}$, and, by assumption $\beta_{\mathrm{GB}}=0, \beta_{\mathrm{GG}}=1$.

\section{Estimating $\alpha$ 's and $G_{I}$ for Continuous Analogue Measurements}

In this case the tested property $x$ of the product is continuous. An example is a test that involves the measurement of an analogue power level which is stored. The measurement model is that $x$ is normally distributed with mean $\mu$ and variance $\sigma^{2}$. Items are truly good if the property value is in the interval $(L, U)$, otherwise bad, so that $G_{I}=P(L<x<U)$. The measured value of the tested property is $y$. The imperfect test is modelled by assuming that, given $x$, the value of $y$ is normally distributed with a mean $x$ and measurement variance $s^{2}$; that the mean of $y$ is $x$ implies that there is no measurement bias. Standard properties of the normal distribution imply that $y$ is distributed normally with a mean $\mu$ and variance $\sigma^{2}+s^{2}$.

Where the Gaussian distribution is inappropriate, one can find a transform of the data where it is a reasonable approximation. What follows in this section then applies to the transformed data, including a transform of $L$ and $U$. This idea is illustrated by the log transform in Section 4.

Recall from Section 1 that our data are: "one-off" data, being a set of $m$ repeated measurements $z_{1}, \ldots, z_{m}$ on one example of the product, first pass data $y_{1}, \ldots, y_{n}$ on $n$ different examples of the product and the yield $p_{r G}$ on the second pass test (after repair), that is the proportion of units that pass the test. Basic statistical theory on sample sizes can give the values of $m$ and $n$ so that with sufficient confidence the estimates of $\mu, \sigma^{2}$ and $s^{2}$ are estimated to a certain precision $[8,9]$.

The variance in the measurements of the one-off test data is $s^{2}$, and so we estimate $s^{2}$ as the sample variance of the $z_{i}$. The mean and variance of the first pass tests are $\mu$ and $\sigma^{2}+s^{2}$ respectively, so that we estimate $\mu$ and $\sigma^{2}+s^{2}$ by the sample mean and variance of the $y_{i}$. Subtracting the estimate of $\sigma^{2}+s^{2}$ from the estimate of $s^{2}$ gives us an estimate of $\sigma^{2}$. Once we have estimates for $\mu, \sigma^{2}$ and $s^{2}$ then we can use these to estimate $\mathrm{G}_{\mathrm{I}}$ and the $\alpha$ 's.

First, $\mathrm{G}_{\mathrm{I}}$ is given by:

$$
G_{I}=P(L<x<U)=\Phi\left(\frac{U-\mu}{\sigma}\right)-\Phi\left(\frac{L-\mu}{\sigma}\right) .
$$

where $\Phi(z)$ is the standard normal distribution function. By the definition of conditional probability, we have:

$$
\begin{aligned}
\alpha_{G G} & =P(L<y<U \mid L<x<U) \\
& =P(L<y<U, L<x<U) / G_{I} .
\end{aligned}
$$

The numerator of this equation is a probability on the joint distribution of $y$ and $x$, which in this case is a bi- variate normal with mean vector $(\mu, \mu)$ and variance matrix:

$$
\Sigma=\begin{array}{cc}
\sigma^{2}+s^{2} & \sigma^{2} \\
\sigma^{2} & \sigma^{2}
\end{array} .
$$

Given this, we can compute $\alpha_{\mathrm{GG}}$ by using:

$$
\begin{gathered}
P(L<y<U, L<x<U)=P(y<U, x<U)-P(y<L, x<U) \\
-P(y<U, x<L)+P(y<L, x<L) \\
=\Phi_{y, x}(U, U)-\Phi_{y, x}(L, U)-\Phi_{y, x}(U, L)+\Phi_{y, x}(L, L) .
\end{gathered}
$$

where $\Phi_{y, x}(y, x)$ is the bivariate normal cumulative distribution function of $y$ and $x$ with the given mean and variance. We can derive an expression for $\alpha_{\mathrm{BB}}$ in a similar manner:

$\alpha_{B B}=P($ item measured bad $\mid$ item is bad $)$

$$
\begin{aligned}
= & 1-P(\text { item measured good } \mid \text { item is bad }) \\
= & 1-P(L<y<U \mid x<L \text { or } x>U) \\
= & 1-P(L<y<U, x<L \text { or } L<y<U, x>U) \\
& \quad / P(x<L \text { or } x>U) .
\end{aligned}
$$

Letting $p_{1}=\mathrm{P}(L<y<U, x<L$ or $L<y<U, x>U)$ and $p_{2}=\mathrm{P}(x<L$ or $x>U)$, we have $\alpha_{\mathrm{BB}}=1-p_{1} / p_{2}$ where:

$$
\begin{gathered}
p_{1}=\Phi_{y, x}(U, L)+\Phi_{y, x}(L, U)-\Phi_{y, x}(U, U)-\Phi_{y, x}(L, L) \\
+\Phi\left(\frac{U-\mu}{\sqrt{\sigma^{2}+s^{2}}}\right)-\Phi\left(\frac{L-\mu}{\sqrt{\sigma^{2}+s^{2}}}\right)
\end{gathered}
$$

and $p_{2}=\Phi((L-\mu) / \sigma)+1-\Phi((U-\mu) / \sigma)$.

Having estimated $G_{I}$ and the $\alpha$ 's, the result of Section 2 can be used to determine the $\beta$ 's from $p_{r G}$. We note that the above formulae hold if the accept interval is infinite, i.e., either $L=-\infty$ or $U=\infty$.

For example, suppose we have a test with accept limits $\mathrm{L}=-13.5$ and $\mathrm{U}=-10$. We observe a sample variance of the one-off test data set to be 0.0028 , the sample mean and variance of the first pass test to be -11.215 and 0.152 , and a yield of $\mathrm{p}_{\mathrm{rG}}=0.942$ from the second pass test. This gives model parameter estimates: $\mu=-11.215, \mathrm{~s}^{2}=$ 0.0028 and $\sigma^{2}=0.152-0.0028=0.1492$. Substituting these into Equations 3.1, 3.2 and 3.3 we compute: $\mathrm{G}_{\mathrm{I}}=$ $0.97, \alpha_{\mathrm{GG}}=0.995$ and $\alpha_{\mathrm{BB}}=0.896$. Substituting these and $\mathrm{p}_{\mathrm{rG}}$ into Equation 2.4, we obtain $\beta_{\mathrm{BG}}=0.93$.

\section{Binary Result of A Continuous Analogue Measurement}

In many tests there is a continuous property being measured, as in the model of Section 3, but the observation is binary, either good (e.g. $L \leq y \leq U$ ) or bad (e.g. $y<L$ or $y>U$ ). The data $z_{1}, \ldots, z_{m}$ and $y_{1}, \ldots, y_{n}$ of Section 3 are now binary (good/bad) and can be summarized by the yields $p_{\text {one }}$ and $p_{G}$, being the proportion of the $z_{j}$ and $y_{i}$ that are good respectively. In this case the underlying measurement model is the same as Section 3 but the data contain less information than in that case and it is much more difficult to obtain the estimates of the $\mu, \sigma^{2}$ and $\mathrm{s}^{2}$ that we need to compute $\alpha$ 's and $G_{I}$. Here we adopt a strategy of making an assumption about the value of $\mu$. We have different assumptions according to the test in 
question. In all cases, the methods that we present will work only if $p_{\text {one }}$ and $p_{G}$ are neither 0 or 1 (must have observed at least one fail and one pass in each set of tests).

\subsection{Assume that the Population Mean is at the Centre of the Accept Interval}

In certain cases it may be reasonable to assume that $\mu$ $=(L+U) / 2$; because the normal distribution is symmetric about its mean, this is equivalent to assuming that the probability that we measure a value below the lower bound $L$ is the same as the probability that we measure a value above the upper bound $U$. We also assume that the true value of the property of the product used in the oneoff tests is close to $\mu$. This leads to a conservative estimation procedure because it produces the largest estimates of $\sigma^{2}$ and $s^{2}$ consistent with the population mean of the property being in the accept interval $(L, U)$.

From the one-off test, an estimate of $\mathrm{P}(L<y<U \mid x)$ is $p_{\text {one. }}$ Our assumptions lead us to the following:

$p_{\text {one }} \approx P(L<y<U \mid x)$

$=\Phi\left(\frac{0.5(U-L)}{s}\right)-\Phi\left(\frac{0.5(L-U)}{s}\right)=2 \Phi\left(\frac{0.5(U-L)}{s}\right)-1$,

which can be solved to get an estimate for s:

$s=\left[0.5(U-L) / \Phi^{-1}\left(0.5\left(1+p_{\text {one }}\right)\right)\right]^{2}$,

where $\Phi^{-1}$ is the inverse normal distribution function (available from tables or in computing environments, like Excel). Note that if we were to observe the measurement from the one-off tests rather than the yield then we could estimate $s^{2}$ more reasonably from the sample variance, as in Section 3. From the observed yield at every test, an estimate of $\mathrm{P}(L<y<U)$ is $p_{G}$. Our assumptions lead us to the following:

$$
\begin{aligned}
p_{G} & \approx P(L<y<U)=\Phi\left(\frac{0.5(U-L)}{\sqrt{\sigma^{2}+s^{2}}}\right)-\Phi\left(\frac{0.5(L-U)}{\sqrt{\sigma^{2}+s^{2}}}\right) \\
& =2 \Phi\left(\frac{0.5(U-L)}{\sqrt{\sigma^{2}+s^{2}}}\right)-1,
\end{aligned}
$$

which can be solved for $\sigma^{2}$ in the same manner as Equation 4.1. The equations in Sections 2 and 3 can then be used to obtain estimates of the $G_{I}, \alpha$ 's and $\beta$ 's.

For example, suppose we have a test with accept limits $\mathrm{L}=-13.5$ and $\mathrm{U}=-10$. We observe a sample variance of the one-off test to be 0.0028 , but only the yield of the first pass test $\mathrm{p}_{\mathrm{G}}=0.995$, and a yield of $\mathrm{p}_{\mathrm{rG}}=0.942$ from the second pass test. We assume that $\mu=-11.75$ (the midpoint of the accept interval). We can still estimate $\mathrm{s}^{2}=$ 0.0028 . Had we not observed the sample variance of the one-off test but just the yield, we would have used Equation 4.1 to compute an estimate of $\mathrm{s}^{2}$. Then we solve Equation 4.2 to get $\sigma^{2}=0.196$. Substituting these into Equations 3.1, 3.2 and 3.3 we compute: $\mathrm{G}_{\mathrm{I}}=0.995, \alpha_{\mathrm{GG}}=$ 0.999 and $\alpha_{\mathrm{BB}}=0.878$. Substituting these and $\mathrm{p}_{\mathrm{rG}}$ into Equation 2.4, we obtain $\beta_{\mathrm{BG}}=0.81$.

\subsection{The Population Mean is not at the Centre of the Accept Interval}

When it is not reasonable to assume that $\mu=(L+U) / 2$, one can still invoke the assumption that the probability that we measure a value below the lower bound $L$ is the same as the probability that we measure a value above the upper bound $U$. One approach to this is to assume that the data are Gaussian on the log scale, and then make this assumption. The property is then lognormally distributed; the mean and variance of the log of the property are $\mu$ and $\sigma^{2}$. We still assume the Gaussian model for measurement error, also on the log scale. In this case, Equations 4.1 and 4.2 still hold as equations to be solved for $\sigma^{2}$ and $s^{2}$ but with $U$ and $L$ replaced by $\log (L)$ and $\log (U)$, while $\mu=$ $(\log (L)+\log (U)) / 2$. The equations in Sections 2 and 3 can then be used to obtain estimates of the $G_{I}, \alpha$ 's and $\beta$ 's, but again with $L$ and $U$ replaced by $\log (L)$ and $\log (U)$.

\subsection{Approaches when Accept Limit is not Finite}

One common scenario where the assumption $\mu=$ $(L+U) / 2$ cannot be used is if either $L=-\infty$ or $U=\infty$. (An example of such a test is the measurment for reciever sensitivity; as long as it is below a certain thresh-hold, it is recorded as passed.) We advocate the use of the model of Section 4.2, assuming a lognormal model and so $x, y \geq 0$. Clearly this is not a viable model if the data can be both positive and negative. In the case where we can assume $x$, $y \geq 0$ then the most extreme lower limit that is $L=0$. Note that if we have $x, y \leq 0$ then we can still use the model of Section 4.2 by applying it to the negative of the data and accept limits.

In the case where $L=0$ and $U<\infty$ then the spirit of the assumption about the mean of Section 4.2 can still be followed. Setting $\mu=0.5 \log (U)$ (the $\log$ of the midpoint of the accept interval) and $x=\mu$ for the product that is tested for the one-off experiments is a value that gives a conservative estimate of $\mathrm{s}^{2}$ and $\sigma^{2}$, obtained by solving for $s$ :

$p_{\text {one }} \approx P(0<y<U \mid x)=\Phi \frac{0.5 \log (U)}{s}$,

and then for $\sigma$ :

$p_{G} \approx P(0<y<U)=\Phi\left(\frac{0.5 \log (U)}{\sqrt{\sigma^{2}+s^{2}}}\right)$.

In the case where $L>0$ and $U=\infty$, it is more difficult to derive a value of $\mu$ that produces a conservative estimate of $\sigma^{2}$ and $s^{2}$. One approach is to set $\mu=c \log (L)$ and $x=\mu$ for some constant $c>1$ which must be chosen according to how far one believes the mean to be from the lower accept limit. Using this, the analogue of Equation 4.3 is: $p_{\text {one }} \approx P(L<y<\infty \mid x)=\Phi \frac{(c-1) \log (L)}{s}$,

and then for $\sigma$ :

$p_{G} \approx P(L<y<\infty)=\Phi \frac{(c-1) \log (L)}{\sqrt{\sigma^{2}+s^{2}}}$. 
There is a solution to Equations 4.3 to 4.6 if and only if $p_{\text {one }}, p_{G}>0.5$, which is another restriction on the estimation procedure for this specific case. The equations in Sections 2 and 3 can then be used to obtain estimates of the $G_{I}, \alpha$ 's and $\beta$ 's, but again with $L$ and $U$ replaced by $\log (L)$ and $\log (U)$.

We note that some of the assumptions made in this section may be unrealistic and lead to errors in estimation of the $G_{I}, \alpha^{\prime}$ 's and $\beta$ 's, They are a consequence of the scarce data available to estimate the parameters. The contrast between this section and Section 3, where more complete data led to a more straightforward estimation procedure with fewer ad-hoc assumptions, demonstrates the desirability of recording as much data as possible during the test.

\section{Binary Result of Discrete Measurement}

In this case there is a count measurement made on the product. The true value of this count is $x$. This count is made with error, and $y$ is the count that we measure. As with the cases of Section 3 and 4, all we are told is whether the measurement shows it to be good (e.g. $L \leq y$ $\leq U$ ) or bad (e.g. $y<L$ or $y>U$ ); our data are the proportion of the products $p_{G}$ that pass the test in the first round of testing and the proportion $p_{r G}$ that pass from the second round of testing of repaired products.

A common model for count data is Poisson and we adopt that here. The true count $x$ is Poisson distributed with an unknown mean $\lambda$. The true count $x$ is measured with Poisson error; the distribution $p(y \mid x)$ is Poisson with mean $x$. Unconditionally, the distribution of the measurement $\mathrm{y}$ is then a mixture of Poisson distributions. There is only one parameter $\lambda$ in this model for $x$ and $y$ (versus $3-\mu, \sigma^{2}$ and $s^{2}-$ in Sections 3 and 4) and this means that it is unecessary to do any "one-off" experiments. We can estimate $\lambda$ every time we run a test using reasonably tractable equations. However the disadvantage of this model is that the mean of $x$ and $y$ given $x$ are equal to the variance - this is the well known property of the Poisson distribution. While there are physical motivations for its use as a count model (as a limiting form of the binomial distribution), the motivation for its use as an error measurement model for $y$ given $x$ is not as strong, other than that it allows a relatively easy solution to the test parameter estimation problem.

The joint distribution of $x$ and $y$ is:

$$
\begin{aligned}
P(X=x, Y=y) & =P(X=x) P(Y=y \mid X=x) \\
& =\frac{\lambda^{x}}{x !} e^{-\lambda} \frac{x^{y}}{y !} e^{-x} .
\end{aligned}
$$

The marginal probability distribution of $y$ is (summing out $x)$ then:

$$
P(Y=y)={ }_{x=0}^{\infty} \frac{\lambda^{x}}{x !} e^{-\lambda} \frac{x^{y}}{y !} e^{-x} .
$$

An estimate of $\mathrm{P}($ board measured good $)=\mathrm{P}(L \leq y \leq$ $U$ ) is $p_{G}$. Using Equation 5.1, we have:

$$
p_{G}=P(L \leq y \leq U)={ }_{y=L x=0}^{U \infty} \frac{\lambda^{x}}{x !} e^{-\lambda} \frac{x^{y}}{y !} e^{-x} .
$$

Equation 5.2 can be solved numerically for $\lambda$, approximating the infinite sum by an appropriate truncated sum. Once $\lambda$ is estimated, we estimate $G_{I}$ and the $\alpha$ 's in a manner similar to Sections 3 and 4. We have:

$$
G_{I}=P(L \leq x \leq U)={ }_{x=L}^{U} \frac{\lambda^{x}}{x !} e^{-\lambda} .
$$

For $\alpha_{\mathrm{GG}}$, we have:

$$
\begin{aligned}
\alpha_{G G}= & \frac{P(L \leq x \leq U, L \leq y \leq U)}{G_{I}} \\
= & \sum_{x=L}^{U} \sum_{y=L}^{U} \frac{\lambda^{x}}{x !} e^{-\lambda} \frac{x^{y}}{y !} e^{-x} / G_{I} .
\end{aligned}
$$

Then for $\alpha_{\mathrm{BB}}$ we have:

$$
\begin{aligned}
& \alpha_{B B}=1-\frac{P(x<L, L \leq y \leq U)+P(x>U, L \leq y \leq U)}{P(x<L)+P(x>U)} \\
& =1-\frac{\left(\sum_{x=0}^{L-1} \sum_{y=L}^{U} \frac{\chi^{x}}{x !} e^{-\lambda} \frac{x^{y}}{y !} e^{-x}\right)+\left(\sum_{x=U+1}^{\infty} \sum_{y=L}^{U} \frac{\lambda^{x}}{x !} e^{-\lambda} \frac{x^{y}}{y !} e^{-x}\right)}{1-\sum_{x=L}^{U} \frac{\lambda^{x}}{x !} e^{-\lambda}} \text { (Eq5.5) }
\end{aligned}
$$

These estimates also hold in the case that $L=0$ or $U=\infty$.

\section{Fully Binary Testing}

In this final case we have a binary result for the test (either pass of fail) and there is a binary classification of the product (good or bad); the data are not a summary of a continuous or discrete measurement. An example is a test that looks for the presence or absence of a component on a circuit board. In this case, the three sets of data are three proportions. From a one-off experiment where a single example of the product was tested, we observe the proportion of tests $p_{\text {one }}$ that passed. From each time that a test is conducted there is the proportion $p_{G}$ that passed the first pass of testing and the proportion $p_{r G}$ that passed the second pass, following repair. This is the one case where the distinction between the parameters of the statistical model - like $\mu$ or $\lambda-$ and the $G_{I}$ and $\alpha$ 's, is lost: we model the test directly with $\mathrm{G}_{\mathrm{I}}, \alpha$ 's and $\beta_{\mathrm{BG}}$.

We make two assumptions. First, $\alpha_{B G}=\alpha_{G B}$ so the probability that a good product is tested to be bad is the same as the probability that you measure a bad product is tested to be good. Second, if we test a single example many times and the proportion of times that the test is passed is greater than 0.5 then assume that we have a truly good example of the product, while if we test a single example many times and the proportion of times that the test is passed is less than 0.5 then assume that we have a truly bad example. Since $\alpha_{\mathrm{GB}}=1-\alpha_{\mathrm{GG}}$, we are assuming that $\alpha_{\mathrm{BG}}=\alpha_{\mathrm{GB}}$ and $\alpha_{\mathrm{BG}}=1-\alpha_{\mathrm{BB}}$, it is sufficient to figure out $\alpha_{\mathrm{GG}}$. The second assumption is assured with high probability, as long as we have tested the product many times.

If $p_{\text {one }} \geq 0.5$ then we assume that we have a good example under test and hence $p_{\text {one }}$ is an estimate of $\alpha_{\mathrm{GG}}$. Given $\alpha_{\mathrm{GG}}=p_{\text {one }}$, we have from our assumptions that $\alpha_{\mathrm{GB}}$ 
$=1-\alpha_{\mathrm{GG}}=1-p_{\text {one }}, \alpha_{\mathrm{BG}}=\alpha_{\mathrm{GB}}=1-\alpha_{\mathrm{GG}}=1-p_{\text {one }}$ and finally $\alpha_{\mathrm{BB}}=1-\alpha_{\mathrm{BG}}=\alpha_{\mathrm{GG}}=p_{\text {one }}$. If $p_{\text {one }}<0.5$ then we assume that we have a bad example and hence $p_{\text {one }}$ is an estimate of $\alpha_{\mathrm{BG}}$. Given $\alpha_{\mathrm{BG}}=p_{\text {one }}$, we have from our assumptions that $\alpha_{\mathrm{GB}}=\alpha_{\mathrm{BG}}=p_{\text {one }}, \alpha_{\mathrm{GG}}=1-\alpha_{\mathrm{GB}}=1-$ $\alpha_{\mathrm{BG}}=p_{\text {one }}$ and finally $\alpha_{\mathrm{BB}}=1-\alpha_{\mathrm{BG}}=1-p_{\text {one }}$.

By the partition law, $\mathrm{P}($ measured good $)=\alpha_{\mathrm{GG}} \mathrm{G}_{\mathrm{I}}+$ $\alpha_{\mathrm{BG}}\left(1-\mathrm{G}_{\mathrm{I}}\right)$ which is estimated by $p_{G}$. Equating $\mathrm{P}$ (measured good) to $p_{G}$ we arrive at an expression for $G_{I}$ in terms of the $\alpha$ 's that we have just computed: $G_{I}=\left[p_{G}\right.$ $\left.-\alpha_{\mathrm{BG}}\right] /\left[\alpha_{\mathrm{GG}}-\alpha_{\mathrm{BG}}\right]$. We note that this is valid if and only if $p_{\text {one }} \geq p_{G}$. This means that the probability of passing cannot be greater than the probability of a good item passing. While intuitively making sense, random chance in the course of the different experiments that compute $p_{\text {one }}$ and $p_{G}$ could give a result that $p_{\text {one }}<p_{G}$ even though their true values do satisfy the condition. Equation 2.1 then allows the $\beta$ 's to be computed, as in rest of paper.

\section{Small Case Study}

An obvious concern with one of the models discussed here is in Section 4, where we only observe the binary response (pass/fail) of a continuous measurement. The simplest solution is in Section 4.1; the population mean $\mu$ $=(\mathrm{L}+\mathrm{U}) / 2$, the centre of the accept interval $(\mathrm{L}, \mathrm{U})$. Here we describe a small simulation study that shows what happens if this assumption is made but is not true.

We generated 100 sets of data from the continuous model with $\mathrm{L}=8, \mathrm{U}=12$ and "true" parameter values $\mu=$ $10, \mathrm{~s}^{2}=0.01$ and $\sigma^{2}=1$. This gives "true" test parameter values $\mathrm{G}_{\mathrm{I}}=0.9545, \alpha_{\mathrm{GG}}=0.995$ and $\alpha_{\mathrm{BB}}=0.92$. For this study we ignored estimation of $\beta_{\mathrm{BG}}$. Each data set consisted of $m=500$ one-off measurements and $n=500$ first pass measurements.

For each set of data we inputted the yields $p_{\text {one }}$ and $p_{G}$ into the method described in Section 4.1, and estimated $\mathrm{G}_{\mathrm{I}}, \alpha_{\mathrm{GG}}$ and $\alpha_{\mathrm{BB}}$, assuming that $\mu=10$ (the mid-point of the accept interval). So in this case our assumption $\mu=10$ was actually correct. The average estimates over the 100 sets of data were: $\mathrm{G}_{\mathrm{I}}=0.97, \alpha_{\mathrm{GG}}=0.999$ and $\alpha_{\mathrm{BB}}=0.999$. So the method worked reasonably well.

We then repeated this experiment but altered the "true" mean used to generate the 100 data sets to $\mu=11 ; \mathrm{s}^{2}$ and $\sigma^{2}$ were kept the same. This leads to $\mathrm{G}_{\mathrm{I}}=0.95, \alpha_{\mathrm{GG}}=$ 0.987 and $\alpha_{\mathrm{BB}}=0.94$. We ran the method of Section 4.1, still assuming, now incorrectly, that $\mu=10$. The average estimates over 100 sets of data were: $G_{I}=0.226, \alpha_{G G}=$ 1.02 and $\alpha_{B B}=0.9998$. So we see that the estimate of $G_{I}$ is much smaller than the true value, while on average the estimate of $\alpha_{\mathrm{GG}}$ does not make sense (it is a probability, so should be between 0 and 1).

\section{Conclusion}

In this paper we have outlined procedures for estimating the parameters of an imperfect test and repair model given different types of data that are typically available from the testing process. In cases where the dimension of the data are less than that of the model parameters, we have proposed some different assumptions that can be made in order to arrive to parameter estimates. As far as possible these are made so that conservative parameter estimates are made. However we observe that there are scenarios, particularly in the cases of Section 4, where strong assumptions must be made and the study in Section 7 shows that if this assumption is wrong then the estimation can have substantial error.

Clearly the next step in this work is to assess the implications of these assumptions for the estimation, and to derive expressions for the estimation error in terms of the error in the assumptions. If this is done then calculations of appropriate sample sizes, and model assessment methods such as computation of standardized residuals, can be made. This work has also motivated consideration of other statistical approaches that may be able to reduce the number of difficult assumptions that must be made; Bayesian inference techniques appear to offer a good alternative with this property.

\section{Acknowledgements}

The involvement of BLI researchers is supported by a grant from the Industrial Development Agency of Ireland. The involvement of CTVR researchers is supported by Science Foundation Ireland grant 03/CE3/I405.

\section{References}

[1] E. Fisher, S. Fortune, M. Gladstein, S. Goyal, W. Lyons, J. Mosher, and G. Wilfong. Economic Modeling of Global Test Strategy I: Mathematical Models. Bell Labs Technical Journal, 12(1), Apr. 2007, To appear.

[2] E. Fisher, S. Fortune, M. Gladstein, S. Goyal, W. Lyons, J. Mosher, and G. Wilfong. Economic Modeling of Global Test Strategy II: Software System and Examples. Bell Labs Technical Journal, 12(1), Apr. 2007, To appear.

[3] B. Gluss. An optimum policy for determining a fault in a complex system. Operations Research, 7:464-477, 1959.

[4] J. A. Nachlas, S. R. Loney, and B. A. Binney. Diagnosticstrategy selection for series systems. IEEE Transactions on Reliability, 30(3):273-280, 1990.

[5] C. Dislis, I.D. Dear, J.R. Miles, S.C. Lau, and A.P. Ambler. Cost analysis of test method environments. In Proceedings of International Test Conference, pages 875-883, 1989.

[6] J. Ding, B. S. Greenberg, and H. Matsuo. Repetitive testing strategies when testing process is imperfect. Management Science, 40(10):1367-1378, 1998.

[7] T. Trichy, P. Sandborn, R. Raghavan, and S. Sahasrabudhe. A New Test/Diagnosis/Rework Model for Use in Technical Cost Modeling of Electronic Systems Assembly. In Proceedings of ITC 2001, pages 1106-1117, 2001 IEEE.

[8] Walpole, R.E. and Myers, R.H. Probability and Statistics for Engineers and Scientists, 4th edition. McMillan, 1989.

[9] S.P. Wilson, B.E. Flood, S. Goyal, J. Mosher, J. O’Brien, and R. Kennedy. Parameter Estimation for Realistic Test \& Repair Models. Bell Labs Technical Memorandum, 2006. 